\title{
Prognostic Significance of the L3 Skeletal Muscle Index and Advanced Lung Cancer Inflammation Index in Elderly Patients with Esophageal Cancer
}

This article was published in the following Dove Press journal: Cancer Management and Research

\author{
Xiang $\operatorname{Tan}^{1, *}$ \\ Huajian Peng ${ }^{\prime * *}$ \\ Peixin $\mathrm{Gu}^{2}$ \\ Mingwu Chen' \\ Yongyong Wang' \\ 'Department of Thoracic Surgery, The \\ First Affiliated Hospital of Guangxi \\ Medical University, Nanning, 53002I, \\ Guangxi Zhuang Autonomous Region, \\ People's Republic of China; ${ }^{2}$ Department \\ of Neurology, The First Affiliated \\ Hospital of Guangxi Medical University, \\ Nanning, People's Republic of China
}

*These authors contributed equally to this work

Correspondence: Mingwu Chen; Yongyong Wang

Department of Thoracic Surgery, The First Affiliated Hospital of Guangxi Medical

University, Shuang Yong Road 6, Nanning, 53002I, Guangxi Zhuang Autonomous

Region, People's Republic of China

Email chen535@I26.com;

wangyongyong@stu.gxmu.edu.cn
Objective: To evaluate the correlation between the advanced lung cancer inflammation index (ALI) and the L3 skeletal muscle index (L3SMI) and their prognostic value in elderly patients with esophageal cancer (EC).

Materials and Methods: The clinical data of 158 elderly patients with EC were collected retrospectively. The L3SMI measures the area of skeletal muscle at the level of the third lumbar (L3) vertebra using computed tomography (CT). A high L3SMI and low L3SMI group were created using sex-based quartiles. The ALI, prognostic nutrition index (PNI), and geriatric nutrition risk index (GNRI) were calculated according to standard laboratory protocols.

Results: The CT diagnostic criteria for senile sarcopenia in South China are height $\leq 32.96 \mathrm{~cm}^{2} /$ $\mathrm{m}^{2}$ for females and height $\leq 35.4 \mathrm{~cm}^{2} / \mathrm{m}^{2}$ for males. The logistic regression analysis showed that a low L3SMI was significantly associated with a low ALI. Survival analysis revealed EC patients with a low L3SMI and a low ALI had poorer overall survival (OS) than patients with a high L3SMI and a high ALI. Univariate and multivariate Cox analyses showed that the L3SMI and ALI were independent predictors of EC prognosis in elderly individuals.

Conclusion: There was a significant correlation between the PNI, GNRI, ALI, and L3SMI. Overall, our findings show the L3SMI and ALI are clinical indicators that can potentially be used to independently predict the prognosis of elderly EC patients and display good predictive value. Keywords: esophageal cancer, sarcopenia, L3 skeletal muscle index, survival, advanced lung cancer inflammation index

\section{Introduction}

Esophageal cancer (EC) is a tumor with the seventh and sixth highest morbidity and mortality rates worldwide, respectively. ${ }^{1}$ With the aging population and the acceleration of population growth, the occurrence of EC has increased. ${ }^{2} \mathrm{EC}$ is primarily divided into squamous cell carcinoma and adenocarcinoma. In East Asia, EC cases are primarily squamous cell carcinoma. The early symptoms of EC are not typical and because EC is aggressive and progresses rapidly, ${ }^{3}$ most patients are at an advanced stage when they are first diagnosed. ${ }^{4,5}$ Esophageal obstruction caused by the progressive growth of EC makes eating difficult for EC patients, which in turn leads to malnutrition and even cachexia. ${ }^{6}$ Many studies have focused on the nutritional status of cancer patients. The geriatric nutritional risk index (GNRI) is a simplified screening parameter calculated based on serum albumin and body weight that can be used to assess nutrition-related risks. ${ }^{7}$ The prognostic nutritional index (PNI) is calculated using the serum albumin concentration and the total 
number of peripheral blood lymphocytes. ${ }^{8}$ The PNI reflects the nutritional and immune status of patients. With the increasing aging population, the number of elderly EC patients is growing. Owing to the heterogeneity of the health status of the elderly, EC patients in this age group lack accurate prognostic predictors. The treatment of EC is still managed using a comprehensive strategy based on surgical intervention. ${ }^{9}$ With improvements in diagnosis and treatment technologies, the prognosis of EC has also improved, but the prognosis of EC patients is still poor. ${ }^{5,10}$ Therefore, exploring reliable indicators for predicting patient survival can help to identify patients with poor prognoses and allow the early adoption of better prevention and treatment methods and reduce mortality.

Skeletal muscle not only plays a role during exercise or movement but also participates in complex immune and inflammatory processes of the body by secreting a variety of cytokines. Hence, some people refer to it as a "secretory organ". ${ }^{11}$ Sarcopenia was first proposed by Rosenberg as the gradual loss of skeletal muscle mass associated with aging. ${ }^{12}$ The L3 skeletal muscle index (L3SMI) assessed using computed tomography (CT) is the primary method for diagnosing sarcopenia. In recent years, sarcopenia has been confirmed to be significantly related to malnutrition and the poor prognosis of various malignant tumors. ${ }^{13-16}$ The commonly used diagnostic criteria for sarcopenia have been primarily formulated by European and American specialists, although there are obvious ethnic differences among Asian, European, and American populations. $^{17,18}$ Thus, the diagnostic criteria in Europe and America may not be applicable to Asian populations. In addition, given to the features of the elderly population, the current diagnostic criteria are not suitable for elderly Asian individuals.

An increasing number of studies have confirmed that tumor-related inflammation plays a vital role in the occurrence and development of tumors. ${ }^{19}$ Recently, many studies have shown that a new inflammation-related index, the advanced lung cancer inflammation index (ALI), can be used to evaluate the prognosis of various malignant tumors, such as non-small-cell lung cancer, ${ }^{20}$ small-cell lung cancer, ${ }^{21}$ and diffuse large B cell lymphoma. ${ }^{22}$ The ALI was first used to assess the prognosis of lung cancer patients. However, researchers have found that the ALI is also a good independent prognostic indicator in head and neck and gastrointestinal tumors. The ALI is associated with poor prognosis in $\mathrm{EC},{ }^{23}$ head and neck squamous cell carcinoma, $^{24}$ and in colorectal cancer. ${ }^{25,26}$
The purpose of this study was to establish the L3SMI cut-off value to be used for diagnosis of sarcopenia in elderly EC patients in southern China (Guangxi Province). In addition, we explored the relationship between the L3SMI and PNI, GNRI, and ALI and their prognostic value in elderly EC patients.

\section{Materials and Methods Study Population}

Data on 189 elderly EC patients who underwent surgical treatment at the First Affiliated Hospital of Guangxi Medical University (Nanning, China) from September 2013 to July 2018 were collected. The inclusion criteria were as follows: (a) EC diagnosis confirmed by pathology; (b) radical esophageal cancer surgery performed; (c) no radiotherapy or chemotherapy conducted prior to surgery; (d) patients aged $\geq 65$ years; ${ }^{27}$ and (e) patients with complete clinical and follow-up data. We used the 8th edition of the American Joint Committee on Cancer (AJCC) TNM grading system to stage patients after surgery. The ethics committee of the First Affiliated Hospital of the Guangxi Medical College approved the study (approval number: 2021(KY-E-046)). All patients in this study provided written informed consent. This study was performed in accordance with the Declaration of Helsinki.

\section{Data Collection}

The clinical data collected from patients was as follows: sex, age, height, and weight. The preoperative laboratory serological examinations of all patients were completed within 1 week prior to surgery. The primary preoperative laboratory serological tests included assessment of lymphocyte counts, neutrophils, and albumin levels. The neutrophil/lymphocyte ratio (NLR) was defined as the neutrophil count divided by the lymphocyte count. Hypoalbuminemia was defined as an albumin level $<35$ $\mathrm{g} / \mathrm{L}$. According to the median NLR, patients were divided into two subgroups: high NLR and low NLR values. The body mass index (BMI) was calculated using the square of weight $(\mathrm{kg}) /$ height $\left(\mathrm{m}^{2}\right)$. BMI values were divided into three subgroups: low $<18.5$, normal $=18.5-24$, and high $\geq 24$. $^{28}$ The PNI was calculated using the following formula: $10 \times$ serum albumin value $(\mathrm{g} / \mathrm{dL})+0.005 \times$ total lymphocyte count in the peripheral blood $\left(\right.$ per $\left.\mathrm{mm}^{3}\right) .{ }^{8}$ The GNRI was calculated as follows: GNRI $=1.489 \times$ serum albumin $(\mathrm{g} / \mathrm{dL})+41.7 \times$ weight/ideal body weight. ${ }^{7}$ The 
ALI was defined as BMI $\left(\mathrm{kg} / \mathrm{m}^{2}\right) \times$ albumin $(\mathrm{g} / \mathrm{dL}) / \mathrm{NLR}$. Time-dependent receiver operating characteristic (ROC) curves were plotted to establish the cutoff values for the GNRI, PNI, and ALI and to establish the high/low GNRI, high/low PNI, and high/low ALI subgroups.

\section{Skeletal Muscle Tissue Measurement}

Enhanced CT imaging of the abdomen of patients the week before treatment was used to collect two consecutive images at the L3 level. The L3 skeletal muscles included the paraspinal, psoas major, rectus abdominis, internal oblique, external oblique, and transverse abdominal muscles. ${ }^{18}$ The L3SMI was calculated as the square of skeletal muscle area $\left(\mathrm{cm}^{2}\right) /$ height $\left(\mathrm{m}^{2}\right)$.

\section{Follow-Up}

All patients in the cohort received telephone or outpatient follow-up after surgery. The deadline for follow-up was 1 January 2021. Overall survival (OS) was defined as the time from surgery to death or the last follow-up.

\section{Statistical Analysis}

All the statistical analyses were performed using SPSS version 19.0. GraphPad Prism version 5.0 and $R$ version 4.0.2 were used to draw the graphs. Time-dependent ROC curves were plotted to establish the cutoff values for the GNRI, PNI, and ALI. The correlations between the L3SMI, ALI, clinicopathological data, and nutritional risk, and the correlations between the L3SMI and ALI and its components were analyzed using a logistic regression. Kaplan-Meier analysis was used to construct a survival curve, and the Log rank test was used for comparisons. The hazard ratios (HRs) and $95 \%$ confidence intervals (CIs) were calculated using univariate and multivariate Cox proportional hazards regression models to evaluate the relationship between the L3SMI and the ALI and prognosis. The important factors in the multivariate Cox analysis were used to construct a nomogram, the coordination index ( $\mathrm{C}$ index), and a calibration curve were used to evaluate the predictive accuracy of the nomogram. A P-value $<0.05$ was considered to indicate statistical significance.

\section{Results}

\section{Patient Characteristics}

A total of 189 elderly EC patients were enrolled from September 2013 to July 2018, of which 10 patients did not have preoperative CT images available, 8 patients did not have complete clinical data, and 13 patients were lost to follow-up. The clinical characteristics of the patients are shown in Table 1. The median age of the patients was 69.5 years (range 65-86 years; mean age $70.7 \pm 4.49$ years). According to the ROC curve (Figure 1), the cutoff value, sensitivity, specificity, and area under the ROC curve (AUC) values for the GNRI and PNI of the 158 elderly EC patients were determined. The cutoff, sensitivity, specificity, and AUC values of the GNRI were 96.60, 60.8\%, $62.0 \%$, and $0.602(95 \% \mathrm{CI}=0.513-0.691)$, respectively. The cutoff, sensitivity, specificity, and AUC values of the PNI were $45.35,75.9 \%, 44.3 \%$, and $0.606(95 \% \mathrm{CI}=$ 0.517-0.694), respectively. The cutoff, sensitivity, specificity, and AUC values of the ALI were 31.24, 62.0\%, $63.3 \%$ and $0.638(95 \% \mathrm{CI}=0.552-0.725)$, respectively.

\section{L3SMI Grouping of the Elderly EC}

\section{Patients}

The L3SMI was divided into four sex-based quartiles: G1, G2, G3, and G4. The Kaplan-Meier survival curves showed that the OS of the G1 group was significantly worse than that of the G2, G3, and G4 groups (Figure 2), and the survival curve of the G1 group was significantly separated from that of the other groups. Thus, the G1 group cutoff appeared more suitable as a threshold for elderly EC sarcopenia. Therefore, based on the above analysis, the 158 elderly EC patients were divided into high and low L3SMI groups. The G2, G3, and G4 groups were combined and represented the high L3SMI group, and the G1 group represented the low L3SMI group.

\section{Relationship Between the L3SMI and ALI and Clinical Pathological Characteristics of the Patients}

A logistic regression analysis was conducted to determine the correlations between patient clinical and pathological characteristics and the L3SMI and ALI (Table 2). ALI and sex $(\mathrm{P}=0.009 ; \mathrm{OR}=0.349,95 \% \mathrm{CI}=0.158-0.771), \mathrm{GNRI}$ $(\mathrm{P}<0.001 ; \mathrm{OR}=8.468,95 \% \mathrm{CI}=3.943-18.186), \mathrm{PNI}$ $(\mathrm{P}=0.001 ; \mathrm{OR}=3.628,95 \% \mathrm{CI}=1.650-7.976)$, and tumor size $(\mathrm{P}=0.035$; $\mathrm{OR}=0.488,95 \% \mathrm{CI}=0.251-0.949)$ were significantly correlated. L3SMI and lymphatic metastasis $(\mathrm{P}=0.035, \mathrm{OR}=0.446,95 \% \mathrm{CI}=0.210-0.946)$, cancer embolus ( $\mathrm{P}=0.004 ; \mathrm{OR}=0.307,95 \% \mathrm{CI}=0.137-0.688)$, tumor depth $(\mathrm{P}=0.006, \mathrm{OR}=0.310,95 \% \mathrm{CI}=0.135-0.711)$, GNRI $(\mathrm{P}<0.001, \mathrm{OR}=5.992,95 \% \mathrm{CI}=2.445-14.685)$, and 
Table I Baseline Characteristics of Patients and Tumors

\begin{tabular}{|c|c|c|}
\hline Characteristics & $\mathbf{n}$ & $\%$ \\
\hline \multicolumn{3}{|l|}{ Age } \\
\hline$\leq 70$ & 84 & $53.16 \%$ \\
\hline$>70$ & 74 & $46.84 \%$ \\
\hline \multicolumn{3}{|l|}{ Gender } \\
\hline Female & 32 & $20.25 \%$ \\
\hline Male & 126 & $79.75 \%$ \\
\hline \multicolumn{3}{|l|}{ BMI } \\
\hline Low & 39 & $24.68 \%$ \\
\hline Normal & 99 & $62.66 \%$ \\
\hline High & 20 & $12.66 \%$ \\
\hline \multicolumn{3}{|l|}{ Cancer embolus } \\
\hline No & 123 & $77.85 \%$ \\
\hline Yes & 35 & $22.15 \%$ \\
\hline \multicolumn{3}{|l|}{ Perineural invasion } \\
\hline No & $14 \mid$ & $89.24 \%$ \\
\hline Yes & 17 & $10.76 \%$ \\
\hline \multicolumn{3}{|l|}{ Smoke } \\
\hline No & 96 & $60.76 \%$ \\
\hline Yes & 62 & $39.24 \%$ \\
\hline \multicolumn{3}{|l|}{ Alcohol drinking } \\
\hline No & 88 & $55.70 \%$ \\
\hline Yes & 70 & $44.30 \%$ \\
\hline \multicolumn{3}{|l|}{ Lymphatic metastasis } \\
\hline No & 82 & $51.90 \%$ \\
\hline Yes & 76 & $48.10 \%$ \\
\hline \multicolumn{3}{|l|}{ Differentiation } \\
\hline Poor & 41 & $25.95 \%$ \\
\hline Moderately & 66 & $41.77 \%$ \\
\hline Well & 51 & $32.28 \%$ \\
\hline \multicolumn{3}{|l|}{ Tumor depth } \\
\hline pTI & 20 & $12.66 \%$ \\
\hline $\mathrm{pT} 2$ & 49 & $31.01 \%$ \\
\hline $\mathrm{pT} 3$ & 86 & $54.43 \%$ \\
\hline $\mathrm{pT} 4$ & 3 & $1.90 \%$ \\
\hline \multicolumn{3}{|l|}{ Tumor location } \\
\hline Upper & 10 & $6.33 \%$ \\
\hline Middle & 89 & $56.33 \%$ \\
\hline Lower & 59 & $37.34 \%$ \\
\hline \multicolumn{3}{|l|}{ Pathology } \\
\hline Squamous cell carcinoma & 148 & $93.67 \%$ \\
\hline Adenocarcinoma & 10 & $6.33 \%$ \\
\hline \multicolumn{3}{|l|}{ Tumor size } \\
\hline$\leq 4 \mathrm{~cm}$ & 82 & $51.90 \%$ \\
\hline$>4 \mathrm{~cm}$ & 76 & $48.10 \%$ \\
\hline
\end{tabular}

Abbreviation: BMI, body mass index.

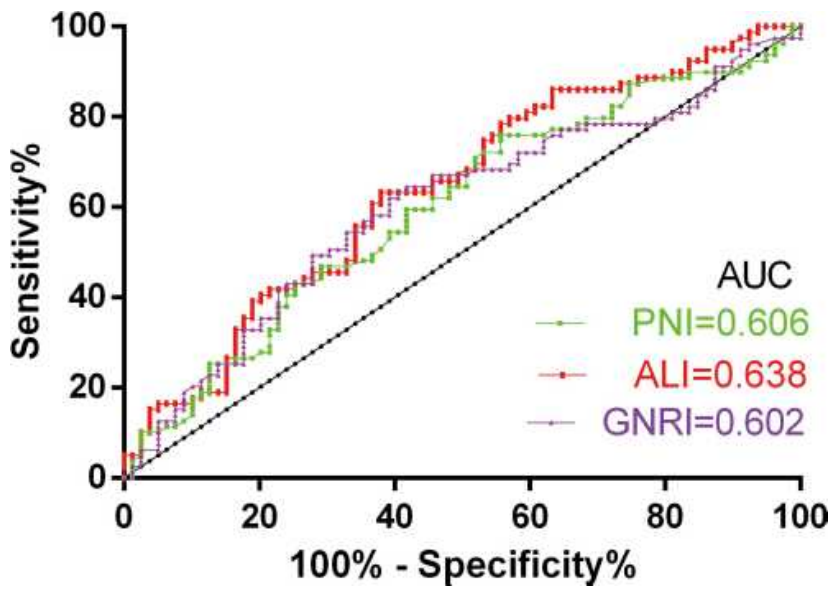

Figure I The receiver-operating characteristic (ROC) curves for GNRI, ALI, and PNI.

Abbreviations: AUC, area under the curve; ALI, advanced lung cancer inflammation index; GNRI, geriatric nutritional risk index; PNI, prognostic nutritional index.

PNI $(\mathrm{P}=0.007, \mathrm{OR}=2.810,95 \% \mathrm{CI}=1.327-5.952)$ were all significantly related.

\section{Relationships Between the L3SMI and ALI and Its Components}

The relationships between the L3SMI and ALI and its components (Table 3) were explored. The L3SMI was significantly related to the ALI $(\mathrm{P}=0.004, \mathrm{OR}=3.942$, 95\% CI $=1.534-10.133)$. The relationship between the L3SMI and the ALI components was further explored. The L3SMI was significantly correlated with the BMI (P $=0.005)$ and NLR $(\mathrm{P}=0.008, \mathrm{OR}=0.185,95 \% \mathrm{CI}=$ 0.054-0.639).

\section{Survival Analysis and Risk Factors for OS}

As of 1 January 2021, a total of 79 patients had died. According to the Kaplan-Meier analysis, patients with a low L3SMI had significantly worse OS than those with a high L3SMI $(\mathrm{P}<0.0001)$ (Figure 3A). Patients with a low ALI had significantly worse OS than those with a high ALI $(\mathrm{P}=0.001)$ (Figure 3B). To evaluate the combined prognostic value of the L3SMI and ALI, 158 elderly EC patients were divided into four subgroups according to combinations of L3SMI and ALI values: low L3SMI/low ALI, low L3SMI/high ALI, high L3SMI/low ALI, and high L3SMI/high ALI. Compared with the other groups, the prognosis of the high L3SMI/ high ALI group was significantly better $(\mathrm{P}<0.0001)$ (Figure 4). Univariate and multivariate Cox analyses were used to identify independent prognostic factors 


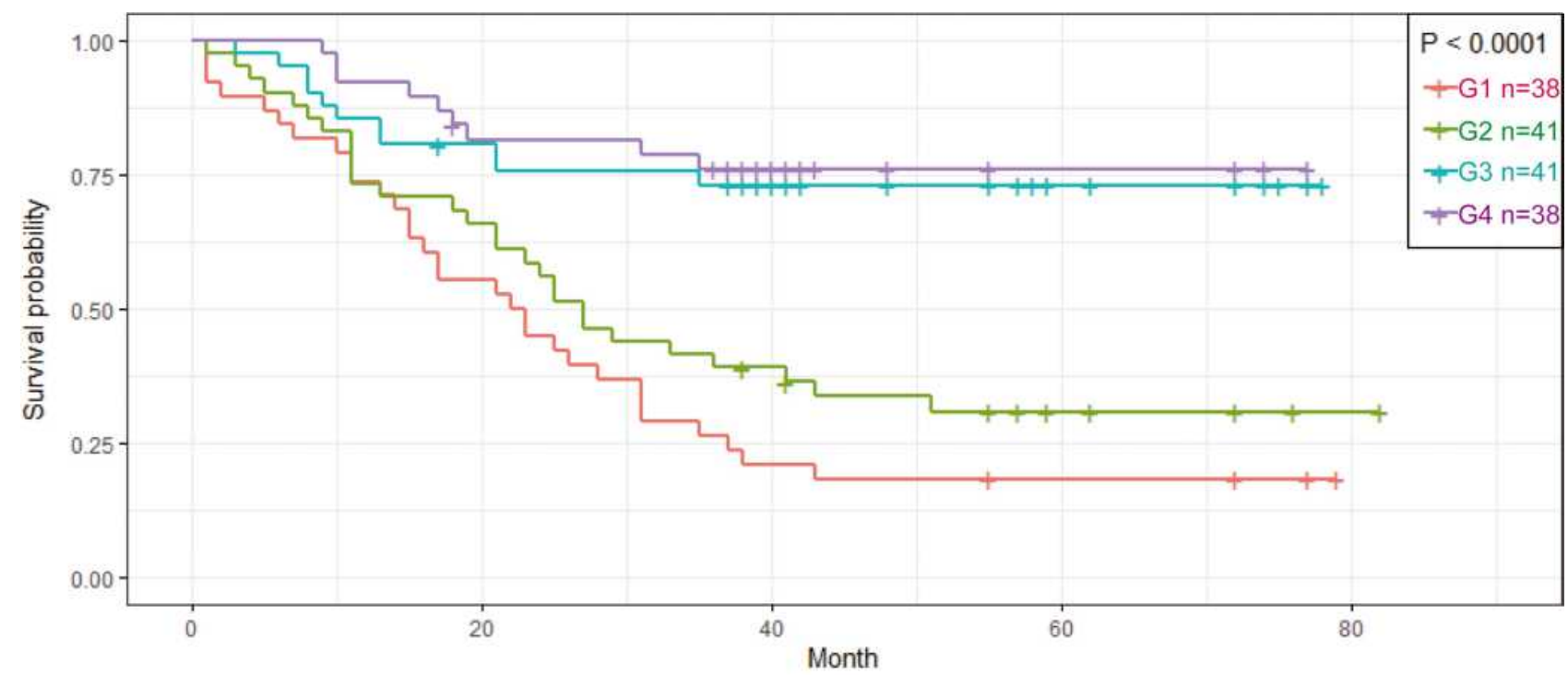

\begin{tabular}{|c|c|c|c|c|c|c|}
\hline \multirow{6}{*}{ 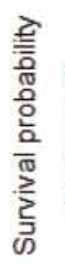 } & & \multicolumn{5}{|c|}{ Number at risk: $n(\%)$} \\
\hline & G1 & $38(100)$ & $21(55)$ & $8(21)$ & $5(13)$ & $0(0)$ \\
\hline & G2 & $41(100)$ & $27(66)$ & $15(37)$ & $5(12)$ & 1 (2) \\
\hline & G3 & $41(100)$ & $32(78)$ & 25 (61) & 7 (17) & $0(0)$ \\
\hline & G4 & $38(100)$ & $30(79)$ & 22 (58) & 4 (11) & $0(0)$ \\
\hline & & 0 & 20 & 40 & 60 & 80 \\
\hline
\end{tabular}

Figure 2 Kaplan-Meier survival curve of the sex-based quartile method (GI-4) of L3SMI in EC patients. Abbreviation: L3SMI, L3 skeletal muscle index.

for the elderly EC patients (Table 4). The univariate Cox analysis indicated that lymphatic metastasis $(\mathrm{P}<0.001$; $\mathrm{HR}=2.756,95 \% \mathrm{CI}=1.728-4.396$ ), cancer embolus $(\mathrm{P}<0.001 ; \mathrm{HR}=2.564,95 \% \mathrm{CI}=1.589-4.137)$, tumor differentiation $(\mathrm{P}=0.003, \quad \mathrm{HR}=2.042, \quad 95 \%$ $\mathrm{CI}=1.281-3.256)$, tumor depth $(\mathrm{P}=0.005$; $\mathrm{HR}=1.961,95 \% \mathrm{CI}=1.225-3.139)$, L3SMI $(\mathrm{P}<$ $0.001 ; \mathrm{HR}=0.366,95 \% \mathrm{CI}=0.232-0.577)$, GNRI $(\mathrm{P}$ $=0.007 ; \mathrm{HR}=0.535,95 \% \mathrm{CI}=0.338-0.845), \mathrm{PNI}(\mathrm{P}=$ $0.009, \mathrm{HR}=0.555,95 \% \mathrm{CI}=0.356-0.865)$, and ALI $(\mathrm{P}$ $=0.002, \mathrm{HR}=0.434,95 \% \mathrm{CI}=0.256-0.735)$ were significantly associated with OS. A multivariate Cox analysis was performed including factors found to significantly affect $O S$ in the univariate analysis. Lymphatic metastasis $(\mathrm{P}=0.013$; $\mathrm{HR}=1.905,95 \% \mathrm{CI}$ $=1.146-3.166)$, tumor differentiation $(\mathrm{P}=0.025 ; \mathrm{HR}=$ $1.781,95 \% \mathrm{CI}=1.075-2.950), \mathrm{L} 3 \mathrm{SMI}(\mathrm{P}=0.019 ; \mathrm{HR}=$ $0.536,95 \% \mathrm{CI}=0.318-0.903)$, and ALI $(\mathrm{P}=0.037$, HR $=0.537,95 \% \mathrm{CI}=0.300-0.962)$ were independent prognostic factors affecting the OS.

\section{Nomogram Model}

Based on the results of the multivariate Cox analysis, a nomogram was constructed for the prediction of patient prognosis. The results indicated that the most important factor for prognosis was lymphatic metastasis, followed by the L3SMI, ALI, and degree of differentiation (Figure 5). By calculating the score of each factor, the 1- to 5-year OS of the EC patients could be predicted for individual patients. The $\mathrm{C}$ index for predicting the OS was 0.707 . The calibration curve showed that the 5-year OS predictions using the nomogram showed the best agreement with the actual observations (Figure 6).

\section{Discussion}

This study found a significant correlation between the L3SMI and ALI. Moreover, the L3SMI and ALI were identified as independent prognostic factors for elderly EC patients. Using our nomogram, we found that the most important factor affecting prognosis was lymphatic metastasis, followed by L3SMI, ALI, and degree of 
Table 2 The Relationships Between the ALI, L3SMI and Clinicopathological Factors of Elderly EC Patients

\begin{tabular}{|c|c|c|c|c|}
\hline \multirow[t]{2}{*}{ Characteristics } & \multicolumn{2}{|c|}{ ALI } & \multicolumn{2}{|c|}{ L3SMI } \\
\hline & OR(95\% Cl) & $\mathbf{P}$ & OR(95\% Cl) & $\mathbf{P}$ \\
\hline Gender (Male) & $0.349(0.158-0.771)$ & 0.009 & $0.858(0.338-2.177)$ & 0.747 \\
\hline Age $(>70)$ & I.439(0.750-2.763) & 0.274 & $0.736(0.354-1.530)$ & 0.412 \\
\hline Smoke (Yes) & $0.674(0.343-1.328)$ & 0.254 & $0.855(0.407-1.795)$ & 0.678 \\
\hline Alcohol drinking (Yes) & $0.55 \mathrm{I}(0.282-1.077)$ & 0.081 & $0.850(0.409-1.767)$ & 0.663 \\
\hline Lymphatic metastasis (Yes) & $0.685(0.356-1.319)$ & 0.258 & $0.446(0.210-0.946)$ & 0.035 \\
\hline Cancer embolus (Yes) & $0.447(0.188-1.066)$ & 0.069 & $0.307(0.137-0.688)$ & 0.004 \\
\hline Perineural invasion (Yes) & $0.713(0.238-2.138)$ & 0.546 & $0.733(0.24 I-2.234)$ & 0.585 \\
\hline Differentiation (Poor) & $0.63 \mid(0.293-1.358)$ & 0.239 & $0.722(0.325-1.606)$ & 0.425 \\
\hline Tumor depth(pT3+pT4) & I.105(0.573-2.129) & 0.766 & $0.310(0.135-0.711)$ & 0.006 \\
\hline GNRI(>96.6) & $8.468(3.943-18.186)$ & $<0.001$ & $5.992(2.445-14.685)$ & $<0.001$ \\
\hline $\mathrm{PNI}(>45.35)$ & $3.628(1.650-7.976)$ & 0.001 & $2.810(1.327-5.952)$ & 0.007 \\
\hline Tumor size $(>4 \mathrm{~cm})$ & $0.488(0.25 I-0.949)$ & 0.035 & $1.039(0.50 \mathrm{I}-2.158)$ & 0.917 \\
\hline Pathology (Adenocarcinoma) & $0.423(0.087-2.063)$ & 0.287 & $0.287(0.078-1.051)$ & 0.060 \\
\hline Tumor location & & 0.344 & & 0.923 \\
\hline Upper & 1 & & I & \\
\hline Middle & $1.019(0.268-3.868)$ & 0.978 & $0.810(0.159-4.111)$ & 0.799 \\
\hline Lower & $0.607(0.152-2.425)$ & 0.480 & $0.733(0.140-3.844)$ & 0.714 \\
\hline
\end{tabular}

Note: Bold values indicate statistically significant values.

Abbreviations: GNRI, Geriatric Nutritional Risk Index; PNI, prognostic nutritional index; L3SMI, L3 skeletal muscle index; ALI, advanced lung cancer inflammation index; EC, esophageal cancer; OR, odds ratio.

differentiation. In this study, we first proposed possible CT diagnostic criteria to distinguish sarcopenia in elderly EC patients in South China (for females with L3SMI $\leq 32.96 \mathrm{~cm}^{2} / \mathrm{m}^{2}$ and males with $\mathrm{L} 3 \mathrm{SMI} \leq 35.4 \mathrm{~cm}^{2} / \mathrm{m}^{2}$ ). Prior to this study, the diagnostic label for sarcopenia was mainly based on sex-specific cutoff values for the L3SMI (males $\leq 52.4 \mathrm{~cm}^{2} / \mathrm{m}^{2}$, females $\leq 38.5 \mathrm{~cm}^{2} / \mathrm{m}^{2}$ ). ${ }^{18}$ These cutoff values were based on the racial characteristics of Europeans and Americans. However, due to ethnic differences between Asians, Europeans, and Americans,

Table 3 The Relationships Between the L3SMI and ALI (and Its Components) of Elderly EC Patients

\begin{tabular}{|c|c|c|c|c|c|}
\hline \multirow[t]{2}{*}{ Characteristics } & \multirow{2}{*}{$\begin{array}{l}\text { Case } \\
\text { No.(\%) }\end{array}$} & \multicolumn{4}{|c|}{ L3SMI } \\
\hline & & Low(\%) & High(\%) & OR(95\% Cl) & $\mathbf{P}$ \\
\hline \multicolumn{6}{|l|}{ ALI } \\
\hline Low & 101 & 32 & 69 & 1 & \\
\hline High & 57 & 6 & 51 & $3.942(1.534-10.133)$ & 0.004 \\
\hline BMI (Kg/m2) & & & & & 0.005 \\
\hline Low & 39 & 17 & 22 & 1 & \\
\hline Normal & 99 & 19 & 80 & $3.254(1.452-7.290)$ & 0.004 \\
\hline High & 20 & 2 & 18 & $6.955(1.415-34.174)$ & 0.017 \\
\hline \multicolumn{6}{|l|}{ Albumin (g/dl) } \\
\hline Low & 23 & 7 & 16 & 1 & \\
\hline Normal & 135 & 31 & 104 & I.468(0.554-3.889) & 0.440 \\
\hline \multicolumn{6}{|l|}{ NLR } \\
\hline Low & 79 & 13 & 66 & 1 & \\
\hline High & 79 & 25 & 54 & $0.185(0.054-0.639)$ & 0.008 \\
\hline
\end{tabular}

Note: Bold values indicate statistically significant values.

Abbreviations: L3SMI, L3 skeletal muscle index; ALI, advanced lung cancer inflammation index; EC, esophageal cancer; BMI, body mass index; NLR, neutrophil-lymphocyte ratio; OR, odds ratio. 

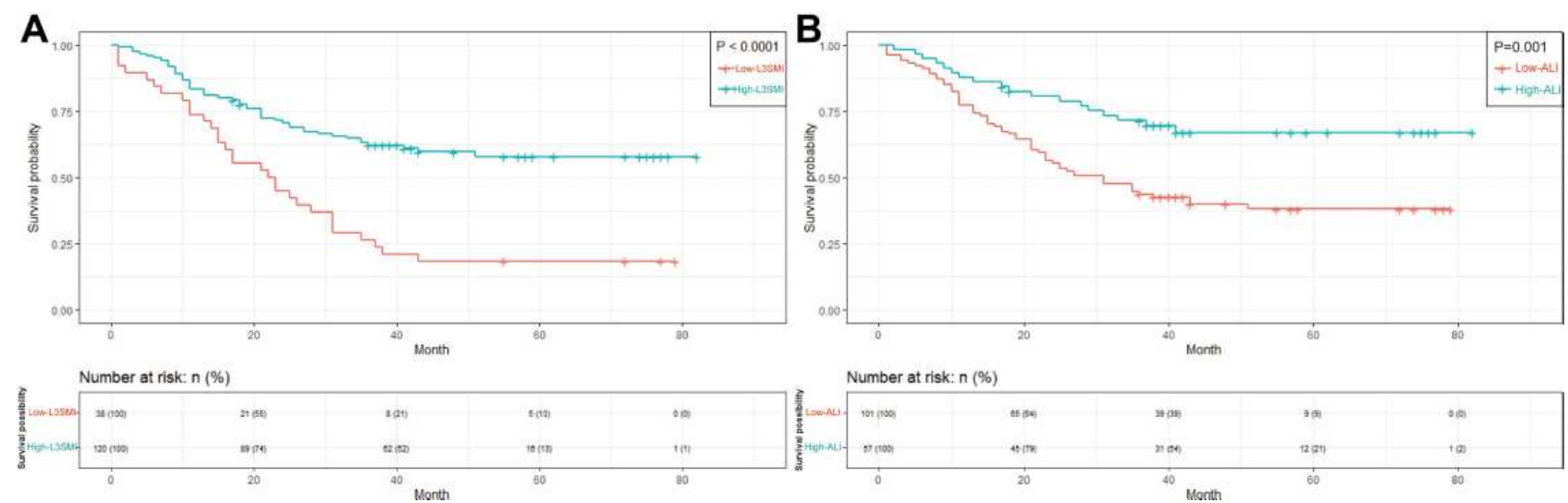

Figure 3 Kaplan-Meier survival curve of overall survival in patients: (A) L3SMI; (B) ALI.

Abbreviations: L3SMI, L3 skeletal muscle index; ALI, advanced lung cancer inflammation index.

there are also differences in the pathological types of EC, especially for the elderly. Therefore, the diagnostic criteria for sarcopenia should be updated.

Many studies have shown that sarcopenia is related to the poor prognosis of many malignant tumors. In this study, it was found that patients with a low L3SMI were more likely to develop lymphatic metastasis and cancer embolus, and were more likely to have an advanced tumor stage than those with a high L3SMI. Further, lower L3SMI was also associated with a lower PNI and GNRI. A total of $60-80 \%$ of patients with EC are often unable to eat due to mechanical obstruction from the tumor, resulting in insufficient nutritional intake. ${ }^{6}$ In addition, elderly individuals are more prone to malnutrition, and their nutritional status is related to the effectiveness of cancer treatment. ${ }^{29,30}$ Poor nutritional status may be related to

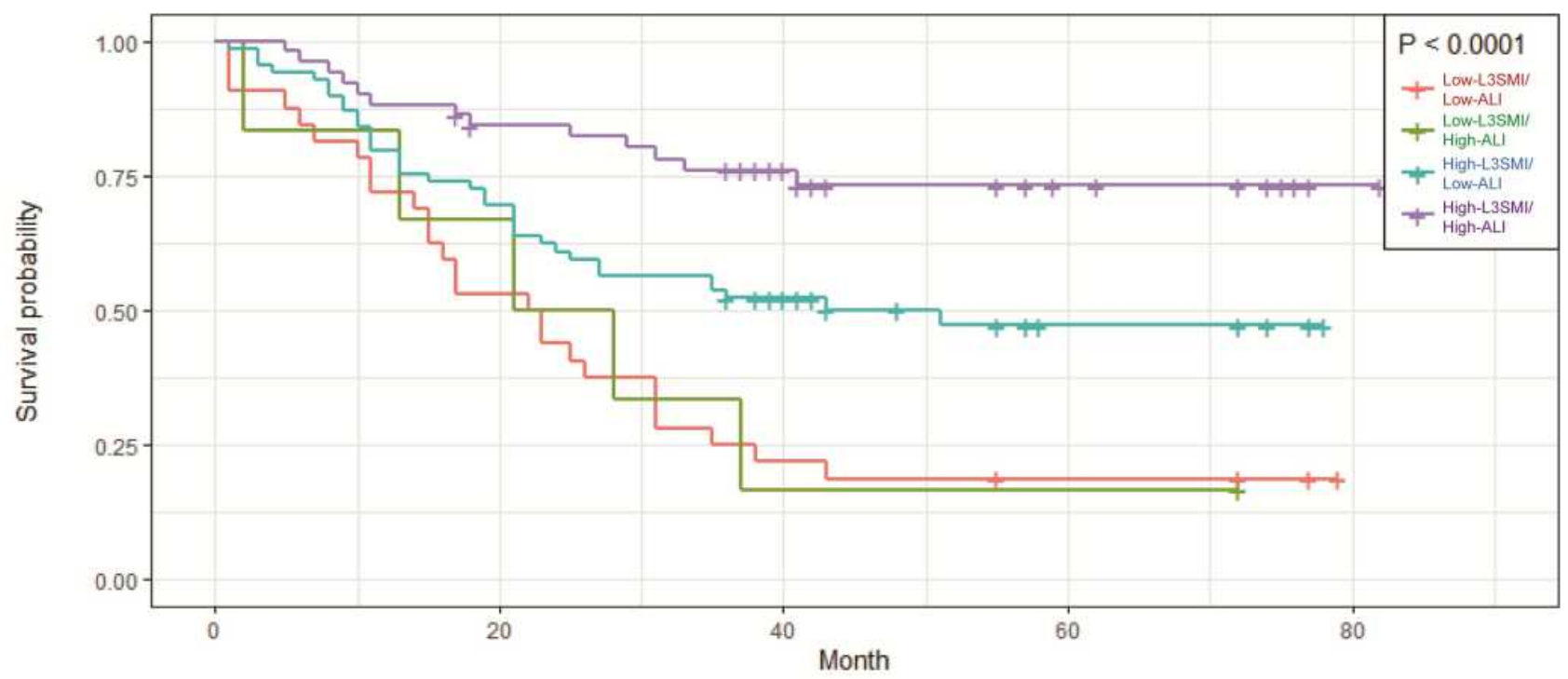

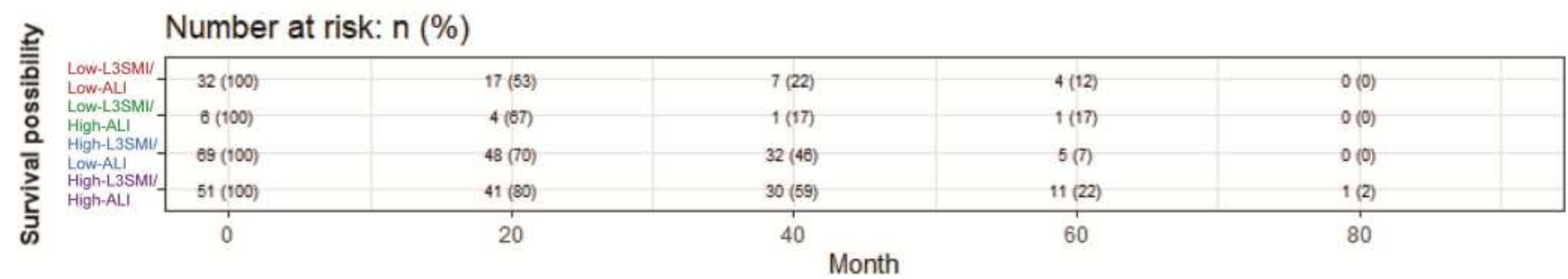

Figure 4 Kaplan-Meier survival curves of patients in subgroups differentiated by L3SMI and ALI. Abbreviations: L3SMI, L3 skeletal muscle index; ALI, advanced lung cancer inflammation index. 
Table 4 Univariate and Multivariate Analyses of Overall Survival (OS) in the Elderly EC Patients

\begin{tabular}{|c|c|c|c|c|}
\hline \multirow[t]{2}{*}{ Characteristics } & \multicolumn{2}{|c|}{ Univariate Analysis } & \multicolumn{2}{|c|}{ Multivariate Analysis } \\
\hline & $\operatorname{HR}(95 \% \mathrm{Cl})$ & $\mathbf{P}$ & HR(95\% Cl) & $\mathbf{P}$ \\
\hline Gender (Male) & $1.029(0.594-1.78 I)$ & 0.920 & & \\
\hline Age $(>70)$ & $1.402(0.902-2.180)$ & 0.133 & & \\
\hline Smoke (Yes) & $1.013(0.645-1.592)$ & 0.956 & & \\
\hline Alcohol drinking (Yes) & $0.842(0.538-1.316)$ & 0.450 & & \\
\hline BMI (Normal+High) & $0.669(0.4|4-1.08|)$ & 0.101 & & \\
\hline Lymphatic metastasis (Yes) & $2.756(1.728-4.396)$ & $<0.001$ & $1.905(1.146-3.166)$ & 0.013 \\
\hline Cancer embolus (Yes) & $2.564(1.589-4.137)$ & $<0.001$ & $1.540(0.900-2.635)$ & 0.115 \\
\hline Perineural invasion (Yes) & $1.334(0.666-2.673)$ & 0.416 & & \\
\hline Differentiation (Poor) & $2.042(I .28 I-3.256)$ & 0.003 & $1.781(1.075-2.950)$ & 0.025 \\
\hline Tumor depth(pT3+pT4) & $1.961(1.225-3.139)$ & 0.005 & $1.184(0.715-1.962)$ & 0.241 \\
\hline L3SMI (high-L3SMI) & $0.366(0.232-0.577)$ & $<0.001$ & $0.536(0.318-0.903)$ & 0.019 \\
\hline GNRI(>96.6) & $0.535(0.338-0.845)$ & 0.007 & $1.017(0.562-1.838)$ & 0.957 \\
\hline $\mathrm{PNI}(>45.35)$ & $0.555(0.356-0.865)$ & 0.009 & $0.807(0.483-1.346)$ & 0.410 \\
\hline Tumor size $(>4 \mathrm{~cm})$ & $1.096(0.705-1.704)$ & 0.683 & & \\
\hline Pathology (Adenocarcinoma) & $1.156(0.467-2.860)$ & 0.754 & & \\
\hline ALI (high) & $0.434(0.256-0.735)$ & 0.002 & $0.537(0.300-0.962)$ & 0.037 \\
\hline Tumor location & & 0.611 & & \\
\hline Upper & I & & & \\
\hline Middle & $0.736(0.3 \mid 4-1.727)$ & 0.482 & & \\
\hline Lower & $0.647(0.268-1.565)$ & 0.334 & & \\
\hline
\end{tabular}

Note: Bold values indicate statistically significant values.

Abbreviations: GNRI, Geriatric Nutritional Risk Index; PNI, prognostic nutritional index; L3SMI, L3 skeletal muscle index; ALI, advanced lung cancer inflammation index; EC, esophageal cancer; HR, hazard ratio.

micro-metastasis and the growth of residual cancer cells, which may lead to a poor prognosis. ${ }^{31}$ Many studies have confirmed that patients with a low L3SMI exhibit a variety of adverse reactions, including increased hospitalization and postoperative rehabilitation costs, ${ }^{32}$ decreased independent living ability, ${ }^{33}$ and increased risk of accidental falls and even fractures caused by decreased skeletal muscle mass. ${ }^{34}$ In addition, skeletal muscle not only plays a role during exercise and movement but also participates in complex immune and inflammatory processes in the body by secreting a variety of cytokines.

Skeletal muscle exerts autocrine, paracrine, and endocrine functions. For example, skeletal muscle can secrete interleukin-6 (IL-6), IL-8, and IL-15. Skeletal muscle can also secrete other inflammatory markers, including tumor necrosis factor- $\alpha$ (TNF- $\alpha$ ) and C-reactive protein (CRP), leading to systemic inflammation. ${ }^{35,36}$ IL-15 can bind natural killer (NK) lymphocytes with high affinity through a variety of signaling pathways and protect NK cells from apoptosis by upregulating $\mathrm{Bcl}-2$ to ensure the normal development and survival of NK cells. ${ }^{37}$ In patients with sarcopenia, the secretion of IL-15 decreases; which leads to a decrease in the anti-tumor ability of the immune system, and may cause lymphatic metastasis, cancer embolus, and tumor progression. ${ }^{38}$

In this study, the ALI was significantly correlated with the GNRI, the PNI, and tumor size. The ALI was then combined with the BMI, albumin, NLR (BMI $\times$ albumin/NLR), and the combination of these factors allowed a better consideration of systemic inflammation and nutritional status. A low ALI score represents a decrease in BMI and serum albumin, and an increase in the NLR, indicated poor nutritional status and high inflammation. An analysis of the L3SMI and ALI (and its components), showed that the L3SMI was significantly related to the ALI, BMI, and NLR. Previous studies have confirmed that BMI is closely related to sarcopenia status, ${ }^{39}$ which is consistent with the results of this study. Inflammation plays a key role in the immune response to pathogens and tissue repair, but it can become chronic and promote the production of reactive oxygen and nitrogen species, stimulate angiogenesis, and cell proliferation, and may ultimately play a key role in the process of cancer. ${ }^{19}$ 
Points

0

10

20

30

40

50

60

70

$80 \quad 90 \quad 100$

ALI

Low

High

L3SMI

Lymphatic metastasis

Differentiation

No

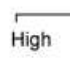

Low

igh

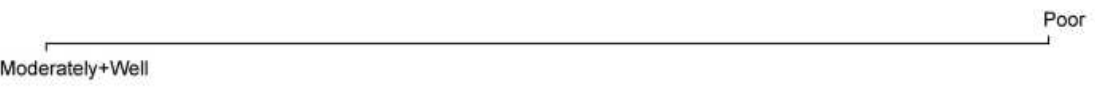

Total Points

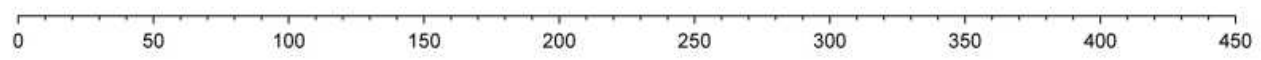

$1-Y e a r$ Survival

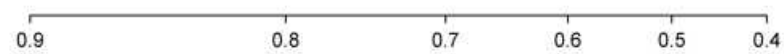

3-Year Survival

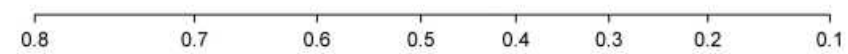

5-Year survival

\begin{tabular}{lllllll}
\hline 0.8 & 0.7 & 1 & 1 & 1 & & \\
\hline & 0.6 & 0.5 & 0.4 & 0.3 & 0.2 & 0.1
\end{tabular}

Figure 5 Nomogram for elderly EC patients. The points identified on the top scale for each independent covariate were added to determine the estimated overall survival and the probability of I-, 3- and 5- year survival.

Abbreviations: L3SMI, L3 skeletal muscle index; ALI, advanced lung cancer inflammation index.

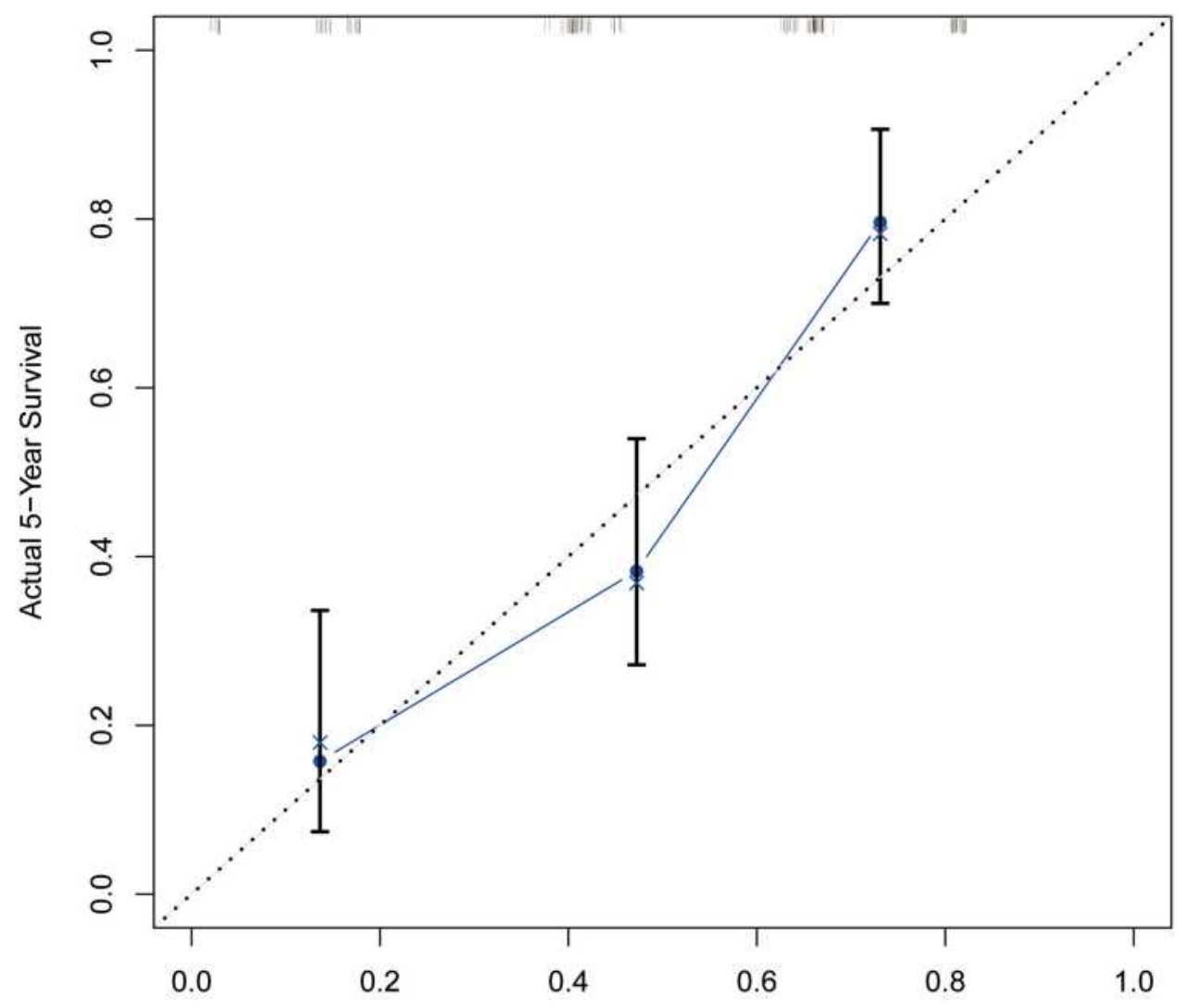

Nomogram-Predicted Probability of 5-Year Survival

Figure 6 The calibration plot for 5-year survival of elderly EC patients. The X-axis presents the predicted probability and the Y-axis shows the actual probability. 
An increase in the NLR indicates an increase in the body's inflammatory response and a decrease in the lymphocyte-mediated anti-tumor response, which can easily cause tumor progression and metastasis. These effects can ultimately lead to a poor prognosis for the patient. ${ }^{40,41}$ The relationship between sarcopenia and systemic inflammation is very tight, which is consistent with these findings. Previous studies have also found that skeletal muscle tissue secretes numerous proinflammatory factors, such as TNF- $\alpha$ and IL-6, which in turn lead to the progression of systemic inflammation. ${ }^{37}$ Systemic inflammation induces the release of proinflammatory cytokines and growth factors, ${ }^{42}$ which in turn exert catabolic effects on host metabolism leading to increased skeletal muscle decomposition. ${ }^{43,44}$ The muscle atrophy caused by inflammation further aggravates systemic inflammation and leads to harmful inflammatory muscle weakness. ${ }^{45}$

This study has certain limitations. First, this study only used skeletal muscle mass to define sarcopenia, ignoring changes in body function. In future, the patient's muscle function should be evaluated in prospective studies. Second, this was a single-center retrospective study with a relatively small sample size; hence, multicenter largescale studies are required to verify our findings. However, diagnostic criteria for sarcopenia in elderly EC patients in South China were also proposed for the first time in this study. Our findings showed that the L3SMI was significantly correlated with the ALI. Early identification of elderly EC patients with poor prognosis and timely intervention can improve the prognosis of elderly EC patients.

\section{Conclusion}

This study was the first to propose diagnostic criteria for sarcopenia specific to elderly patients with EC in South China: an L3SMI $\leq 32.96 \mathrm{~cm}^{2} / \mathrm{m}^{2}$ for females and an L3SMI $\leq 35.4 \mathrm{~cm}^{2} / \mathrm{m}^{2}$ for males. The L3SMI and ALI are significantly related, and both are independent prognostic factors in elderly EC patients. The nomogram considering the ALI and L3SMI provides a scientific basis for identifying patients with poor prognoses.

\section{Acknowledgments}

This work was supported in part by the National Natural Science Foundation of China (81660387) and Development and Application of Medical and Health Appropriate Technology of Guangxi (S201654). Xiang Tan and Huajian Peng are co first authors.

\section{Disclosure}

The authors declare that they have no competing interests.

\section{References}

1. Bray F, Ferlay J, Soerjomataram I, Siegel RL, Torre LA, Jemal A. Global cancer statistics 2018: GLOBOCAN estimates of incidence and mortality worldwide for 36 cancers in 185 countries. CA Cancer J Clin. 2018;68(6):394-424. doi:10.3322/caac.21492

2. Domper Arnal MJ, Ferrandez Arenas A, Lanas Arbeloa A. Esophageal cancer: risk factors, screening and endoscopic treatment in Western and Eastern countries. World J Gastroenterol. 2015;21 (26):7933-7943. doi:10.3748/wjg.v21.i26.7933

3. Morita M, Yoshida R, Ikeda K, et al. Advances in esophageal cancer surgery in Japan: an analysis of 1000 consecutive patients treated at a single institute. Surgery. 2008;143(4):499-508. doi:10.1016/j. surg.2007.12.007

4. Huang FL, Yu SJ. Esophageal cancer: risk factors, genetic association, and treatment. Asian J Surg. 2018;41(3):210-215. doi:10.1016/j. asjsur.2016.10.005

5. Lin Y, Totsuka Y, He Y, et al. Epidemiology of esophageal cancer in Japan and China. J Epidemiol. 2013;23(4):233-242. doi:10.2188/jea. JE20120162

6. Mariette C, De Botton ML, Piessen G. Surgery in esophageal and gastric cancer patients: what is the role for nutrition support in your daily practice? Ann Surg Oncol. 2012;19(7):2128-2134.

7. Bouillanne O, Morineau G, Dupont C, et al. Geriatric nutritional risk index: a new index for evaluating at-risk elderly medical patients. $\mathrm{Am}$ J Clin Nutr. 2005;82(4):777-783. doi:10.1093/ajen/82.4.777

8. Onodera T, Goseki N, Kosaki G. Prognostic nutritional index in gastrointestinal surgery of malnourished cancer patients. Nihon Geka Gakkai Zasshi. 1984;85(9):1001-1005.

9. Ando $\mathrm{N}$, Kato $\mathrm{H}$, Igaki $\mathrm{H}$, et al. A randomized trial comparing postoperative adjuvant chemotherapy with cisplatin and 5-fluorouracil versus preoperative chemotherapy for localized advanced squamous cell carcinoma of the thoracic esophagus (JCOG9907). Ann Surg Oncol. 2012;19(1):68-74. doi:10.1245/ s10434-011-2049-9

10. Peery AF, Crockett SD, Barritt AS, et al. Burden of gastrointestinal, liver, and pancreatic diseases in the United States. Gastroenterology. 2015;149(7):1731-1741 e1733. doi:10.1053/j.gastro.2015.08.045

11. Pedersen BK, Febbraio MA. Muscles, exercise and obesity: skeletal muscle as a secretory organ. Nat Rev Endocrinol. 2012;8(8):457-465. doi:10.1038/nrendo.2012.49

12. Rosenberg IH. Sarcopenia: origins and clinical relevance. J Nutr. 1997;127(5Suppl):990S-991S. doi:10.1093/jn/127.5.990S

13. Yang M, Shen Y, Tan L, Li W. Prognostic value of sarcopenia in lung cancer: a systematic review and meta-analysis. Chest. 2019;156 (1):101-111. doi:10.1016/j.chest.2019.04.115

14. Caan BJ, Meyerhardt JA, Kroenke CH, et al. Explaining the obesity paradox: the association between body composition and colorectal cancer survival (C-SCANS Study). Cancer Epidemiol Biomarkers Prev. 2017;26(7):1008-1015. doi:10.1158/1055-9965.EPI-17-0200

15. Shachar SS, Williams GR, Muss HB, Nishijima TF. Prognostic value of sarcopenia in adults with solid tumours: a meta-analysis and systematic review. Eur $J$ Cancer. 2016;57:58-67. doi:10.1016/j. ejca.2015.12.030

16. Sueda T, Takahasi H, Nishimura J, et al. Impact of low muscularity and myosteatosis on long-term outcome after curative colorectal cancer surgery: a propensity score-matched analysis. Dis Colon Rectum. 2018;61(3):364-374. doi:10.1097/DCR.0000000000000958

17. Lau EM, Lynn HS, Woo JW, Kwok TC, Melton LJ 3rd. Prevalence of and risk factors for sarcopenia in elderly Chinese men and women. J Gerontol A Biol Sci Med Sci. 2005;60(2):213-216. doi:10.1093/ gerona/60.2.213 
18. Prado CM, Lieffers JR, McCargar LJ, et al. Prevalence and clinical implications of sarcopenic obesity in patients with solid tumours of the respiratory and gastrointestinal tracts: a population-based study. Lancet Oncol. 2008;9(7):629-635. doi:10.1016/S1470-2045(08)70153-0

19. Mantovani A, Allavena P, Sica A, Balkwill F. Cancer-related inflammation. Nature. 2008;454(7203):436-444. doi:10.1038/ nature 07205

20. Jafri SH, Shi R, Mills G. Advance lung cancer inflammation index (ALI) at diagnosis is a prognostic marker in patients with metastatic non-small cell lung cancer (NSCLC): a retrospective review. $B M C$ Cancer. 2013;13:158. doi:10.1186/1471-2407-13-158

21. Kim EY, Kim N, Kim YS, et al. Prognostic significance of modified Advanced Lung Cancer Inflammation Index (ALI) in patients with small cell lung cancer_comparison with original ALI. PLoS One. 2016;11(10):e0164056. doi:10.1371/journal.pone.0164056

22. Park YH, Yi HG, Lee MH, Kim CS, Lim JH. Prognostic value of the pretreatment Advanced Lung Cancer Inflammation Index (ALI) in diffuse large B cell lymphoma patients treated with R-CHOP chemotherapy. Acta Haematol. 2017;137(2):76-85. doi:10.1159/ 000452991

23. Feng JF, Huang Y, Chen QX. A new inflammation index is useful for patients with esophageal squamous cell carcinoma. Onco Targets Ther. 2014;7:1811-1815.

24. Hua X, Chen J, Wu Y, Sha J, Han S, Zhu X. Prognostic role of the advanced lung cancer inflammation index in cancer patients: a meta-analysis. World J Surg Oncol. 2019;17(1):177. doi:10.1186/ s12957-019-1725-2

25. Shibutani M, Maeda K, Nagahara H, et al. The prognostic significance of the advanced lung cancer inflammation index in patients with unresectable metastatic colorectal cancer: a retrospective study. BMC Cancer. 2019;19(1):241. doi:10.1186/s12885-019-5468-9

26. Xie H, Huang S, Yuan G, et al. The advanced lung cancer inflammation index predicts short and long-term outcomes in patients with colorectal cancer following surgical resection: a retrospective study. PeerJ. 2020;8:e10100. doi:10.7717/peerj.10100

27. Nakashima Y, Saeki H, Nakanishi R, et al. Assessment of sarcopenia as a predictor of poor outcomes after esophagectomy in elderly patients with esophageal cancer. Ann Surg. 2018;267(6):1100-1104. doi:10.1097/SLA.0000000000002252

28. Wang S, Xie H, Gong Y, et al. The value of L3 skeletal muscle index in evaluating preoperative nutritional risk and long-term prognosis in colorectal cancer patients. Sci Rep. 2020;10(1):8153. doi:10.1038/ s41598-020-65091-0

29. Eo WK, Chang HJ, Suh J, et al. The prognostic nutritional index predicts survival and identifies aggressiveness of gastric cancer. Nutr Cancer. 2015;67(8):1260-1267. doi:10.1080/01635581.2015.1 082112

30. Andreyev HJ, Norman AR, Oates J, Cunningham D. Why do patients with weight loss have a worse outcome when undergoing chemotherapy for gastrointestinal malignancies? Eur J Cancer. 1998;34 (4):503-509.

31. Toyokawa T, Kubo N, Tamura T, et al. The pretreatment Controlling Nutritional Status (CONUT) score is an independent prognostic factor in patients with resectable thoracic esophageal squamous cell carcinoma: results from a retrospective study. BMC Cancer. 2016;16:722. doi:10.1186/s12885-016-2696-0

Cancer Management and Research

Publish your work in this journal

Cancer Management and Research is an international, peer-reviewed open access journal focusing on cancer research and the optimal use of preventative and integrated treatment interventions to achieve improved outcomes, enhanced survival and quality of life for the cancer patient.
32. Mijnarends DM, Luiking YC, Halfens RJG, et al. Muscle, health and costs: a glance at their relationship. J Nutr Health Aging. 2018;22 (7):766-773. doi:10.1007/s12603-018-1058-9

33. Dos Santos L, Cyrino ES, Antunes M, Santos DA, Sardinha LB. Sarcopenia and physical independence in older adults: the independent and synergic role of muscle mass and muscle function. J Cachexia Sarcopenia Muscle. 2017;8(2):245-250. doi:10.1002/ jcsm. 12160

34. Schaap LA, van Schoor NM, Lips P, Visser M. Associations of sarcopenia definitions, and their components, with the incidence of recurrent falling and fractures: the Longitudinal Aging Study Amsterdam. J Gerontol A Biol Sci Med Sci. 2018;73(9):1199-1204. doi:10.1093/gerona/glx245

35. Quinn LS. Interleukin-15: a muscle-derived cytokine regulating fat-to-lean body composition. J Anim Sci. 2008;86(14 Suppl):E75E83. doi:10.2527/jas.2007-0458

36. Schaap LA, Pluijm SM, Deeg DJ, et al. Higher inflammatory marker levels in older persons: associations with 5-year change in muscle mass and muscle strength. J Gerontol A Biol Sci Med Sci. 2009;64 (11):1183-1189. doi:10.1093/gerona/glp097

37. Lutz CT, Quinn LS. Sarcopenia, obesity, and natural killer cell immune senescence in aging: altered cytokine levels as a common mechanism. Aging. 2012;4(8):535-546. doi:10.18632/aging.100482

38. Berger A, Colpitts SJ, Seabrook MSS, et al. Interleukin-15 in cancer immunotherapy: IL-15 receptor complex versus soluble IL-15 in a cancer cell-delivered murine leukemia model. J Immunother Cancer. 2019;7(1):355. doi:10.1186/s40425-019-0777-8

39. Kim KM, Jang HC, Lim S. Differences among skeletal muscle mass indices derived from height-, weight-, and body mass index-adjusted models in assessing sarcopenia. Korean J Intern Med. 2016;31 (4):643-650. doi:10.3904/kjim.2016.015

40. Wang D, DuBois RN. Immunosuppression associated with chronic inflammation in the tumor microenvironment. Carcinogenesis. 2015;36(10):1085-1093. doi:10.1093/carcin/bgv123

41. Cerwenka A, Lanier LL. Natural killer cell memory in infection, inflammation and cancer. Nat Rev Immunol. 2016;16(2):112-123. doi:10.1038/nri.2015.9

42. Feliciano EMC, Kroenke CH, Meyerhardt JA, et al. Association of systemic inflammation and sarcopenia with survival in nonmetastatic colorectal cancer: results from the C SCANS Study. JAMA Oncol. 2017;3(12):e172319. doi:10.1001/jamaoncol.2017.2319

43. Fearon KC, Glass DJ, Guttridge DC. Cancer cachexia: mediators, signaling, and metabolic pathways. Cell Metab. 2012;16(2):153-166. doi:10.1016/j.cmet.2012.06.011

44. Lin JX, Lin JP, Xie JW, et al. Prognostic value and association of sarcopenia and systemic inflammation for patients with gastric cancer following radical gastrectomy. Oncologist. 2019;24(11):e1091e1101. doi:10.1634/theoncologist.2018-0651

45. Kalinkovich A, Livshits G. Sarcopenic obesity or obese sarcopenia: a cross talk between age-associated adipose tissue and skeletal muscle inflammation as a main mechanism of the pathogenesis. Ageing Res Rev. 2017;35:200-221.
The manuscript management system is completely online and includes a very quick and fair peer-review system, which is all easy to use. Visit http://www.dovepress.com/testimonials.php to read real quotes from published authors. 